\title{
The Role of the Lateral Prefrontal Cortex and Anterior Cingulate in Stimulus-Response Association Reversals
}

\author{
Benjamin A. Parris, Ngoc J. Thai, Abdelmalek Benattayallah, \\ Ian R. Summers, and Timothy L. Hodgson
}

\begin{abstract}
Many complex tasks require us to flexibly switch between behavioral rules, associations, and strategies. The prefrontal cerebral cortex is thought to be critical to the performance of such behaviors, although the relative contribution of different components of this structure and associated subcortical regions are not fully understood. We used functional magnetic resonance imaging to measure brain activity during a simple task which required repeated reversals of a rule linking a colored cue and a left/right motor response. Each trial comprised three discrete events separated by variable delay periods. A colored cue instructed which response was to be executed, followed by a go signal which told the subject to execute the response and a feedback instruction which indicated whether to "hold" or "flip" the rule linking the colored cue and response. The design allowed us to determine which brain regions were recruited by the specific demands of
\end{abstract}

\section{INTRODUCTION}

In order for behavior to be adaptive across changing motivational contexts, neural mappings between stimulus and response must be flexible and responsive to changing task demands/goals. Many theorists have suggested that the prefrontal cerebral cortex is critical to such adaptive control (Miller \& Cohen, 2001; Duncan \& Owen, 2000; Duncan, 1999). More precisely, several models postulate that the prefrontal cortex maintains representations that define the appropriate stimulusresponse (SR) mappings to be used for decision making in a given context (Shallice \& Burgess, 1996; Cohen \& Servan-Schreiber, 1992; Baddeley, 1988). However, the role played by different components of this prefrontal network remains the topic of debate. Here we used event-related functional magnetic resonance imaging (fMRI) to examine activity within different prefrontal regions during performance of a simple task, which required participants to repeatedly switch between SR associations.

University of Exeter, Exeter, UK preparing a rule contingent motor response, executing such a response, evaluating the significance of the feedback, and reconfiguring stimulus-response (SR) associations. The results indicate that an increase in neural activity occurs within the anterior cingulate gyrus under conditions in which SR associations are labile. In contrast, lateral frontal regions are activated by unlikely/unexpected perceptual events regardless of their significance for behavior. A network of subcortical structures, including the mediodorsal nucleus of the thalamus and striatum were the only regions showing activity that was exclusively correlated with the neurocognitive demands of reversing SR associations. We conclude that lateral frontal regions act to evaluate the behavioral significance of perceptual events, whereas medial frontal-thalamic circuits are involved in monitoring and reconfiguring SR associations when necessary.

A number of previous imaging studies have examined the brain regions engaged during rule/task switching (Forstmann, Brass, Koch, \& von Cramon, 2005; Derrfuss, Brass, \& von Cramon, 2004; Braver, Reynolds, \& Donaldson, 2003; , Konishi et al., 2002; Kimberg, Aguirre, \& D'Esposito, 2000; MacDonald, Cohen, Stenger, \& Carter, 2000; Carter, Botvinick, \& Cohen, 1999; Konishi, Nakajima, et al., 1999). Many studies report activity in dorsolateral prefrontal regions coincident with the onset of stimuli which instruct the subject which task is to be executed on a given trial. This is consistent with a role for this area in endogenous maintenance of task rules/ goals in advance of task execution (Sakai \& Passingham, 2003; MacDonald et al., 2000; Konishi, Kawazua, et al., 1999). Other studies have claimed a role for ventrolateral frontal regions in the suppression of automatically cued motor responses under conditions of task/rule conflict (Aron, Monsell, Sahakian, \& Robbins, 2004; Swainson et al., 2003; Konishi, Nakajima, et al., 1999), as well as the learning of arbitrary mappings between visual stimuli and motor actions (Passingham, Toni, \& Rushworth, 2000). All of these functions are compatible with a more general role for the lateral prefrontal cortex as a working 
memory buffer, within which the neural representation of current task rules are maintained and updated (Owen, 2000; D'Esposito et al., 1998)

Task switching studies typically also reveal activity in areas of the medial prefrontal cortex, including the anterior cingulate gyrus. One recent combined fMRI and transcranial magnetic stimulation (TMS) study by Rushworth, Hadland, Paus, and Sipila (2002) examined a "motor" switching task, in which subjects switched between two conflicting rules linking two colored shapes with either a left or a right button press. The event of interest was the instruction given to either hold or change rule following runs of correct trials of variable length. Three regions within the medial prefrontal cortex were activated by the instruction to "switch" rules, including the "rostral" and "caudal" cingulate gyrus and the more posterior presupplementary motor area (preSMA). Repetitive TMS applied over the pre-SMA was found to slow reaction times, but only when applied following the signal which instructed a change in rules. One interpretation of this result is that medial prefrontal regions might be important in the process of reconfiguring SR mappings when the task demands it.

It has also been suggested that activity in the medial prefrontal cortex is correlated with generalized arousal during situations of high task demand. Paus (2001) has pointed out that the medial prefrontal cortex receives extensive input from limbic structures such as the amygdala (Kunishi \& Haber, 1994; Barbas \& De Olmos, 1990). It also receives connections from the mediodorsal and midline thalamic nuclei (Shyu, Lin, Sun, Chen, \& Chang, 2004; Morecraft \& Van Hoesen, 1998; Barbas, Henion, \& Dermon, 1991), which have been implicated in modulating general levels of cortical arousal (Montaron \& Buser, 1988). These inputs may provide an important "alerting" signal, which activates the medial prefrontal cortex in order to monitor and resolve conflict in motor output channels (Braver, Barch, Gray, Molfese, \& Snyder, 2001).

The process via which lateral and medial prefrontal regions interact during cognitive tasks is a major unanswered question. Coactivation of the two regions is observed in most cognitively demanding situations (Paus, 2001) and the existence of strong anatomical connections between the anterior cingulate and the dorsolateral prefrontal cortex is well established (Barbas \& Pandya, 1989). One recent study highlighted the importance of interactions between the lateral and medial prefrontal cortex in evaluating the significance of taskrelevant feedback. Gehring and Knight (2000) used event-related potentials (ERPs) to examine how the medially localized error-related negativity component of ERPs is modulated by damage to lateral prefrontal regions in humans. Interestingly, a strong medial negativity was observed following a motor response in patients. However, unlike healthy individuals, the size of this potential was not modulated by whether or not the response was correct or incorrect. The authors proposed, on the basis of this finding, that the lateral prefrontal cortex and the anterior cingulate act together to evaluate the significance of feedback (e.g., correct/ incorrect) for future behavior. Lateral prefrontal regions modulate activity within the anterior cingulate when errors are detected. The medial prefrontal cortex then makes compensatory adjustments to SR mappings via its interactions with subcortical structures and posterior cortical regions (Gehring \& Knight, 2000).

We carried out an event-related fMRI study to examine the contribution of prefrontal cortical regions and associated subcortical structures to adaptive behavioral control. We used a simple rule switching task similar to one which we have previously examined in patients with lateral prefrontal and medial prefrontal cortex damage (Hodgson \& Golding, 2003; Husain, Parton, Hodgson, Mort, \& Rees, 2003). Neurophysiological studies using a very similar task have also revealed the existence of neurons in the lateral prefrontal cortex of monkeys that show activity tuned to particular SR associations (Asaad, Rainer, \& Miller, 1998). In our version of the task, each trial included three discrete events: a colored cue, which instructed which response was to be executed; a go signal, which told the subject to execute the instructed response; and a feedback event, which indicated to the subject whether to "hold" or "flip" the association between the colored cue and response. This allowed us to determine which brain regions were recruited by the instructions to change SR associations, as distinct to those involved in preparing and executing motor behavior in the task. A follow-up experiment addressed the question of whether differences in the pattern of neural activity observed for "flip" relative to "hold" rule trials could be due in part to the perceptual characteristics of the stimuli used to instruct rule changes, rather than the demand to reverse SR mappings per se.

\section{EXPERIMENT 1}

\section{Methods}

\section{Participants}

Twenty-two right-handed volunteers, ranging in age from 21 to 52 years (mean age $=25$ years), gave informed consent to participate in the experiment, which was approved by the School of Psychology Ethics Committee. Thirteen of the participants were women and nine were men.

\section{fMRI Data Acquisition}

Scanning was performed on a 1.5-T Philips Gyroscan magnet at the Peninsula MRI research centre, University of Exeter, UK. A T2*-weighted echo-planar sequence was used $\left(\mathrm{TR}=3000 \mathrm{msec}, \mathrm{TE}=50 \mathrm{msec}\right.$, flip angle $90^{\circ}$, 32 transverse slices, $3.6 \times 3.6 \times 4 \mathrm{~mm}^{3}$, ascending ac- 
quisition). A total of 125 volumes were acquired in each of the four runs per subject. An additional five "dummy" scans were performed at the start of each block prior to the start of the stimulus sequence.

\section{Task and Procedure}

Visual stimuli were presented on a back-projection screen positioned at the foot end of the MRI scanner and viewed via a mirror mounted on the head coil. The screen subtended $16^{\circ}$ of visual arc. Button-press responses and reaction times were measured using two fiber-optic button boxes held in the participants' right and left hands.

Each trial in the experiment began with the presentation of a blue or yellow colored circle (cue event) in the center of the projection screen. Participants were instructed to refrain from making a motor response until the cue dimmed (go event), at which point they pressed either the left or the right hand response key depending upon the current rule mapping (participants were instructed with the initial rule: blue $=$ left, yellow $=$ right $)$. Following another variable delay period, an instruction was presented which indicated to the subject whether to hold or reverse the rule on the following trial (feedback event) (Figure 1). Feedback events were either words ("hold"/flip") or symbolic instructions (happy/sad

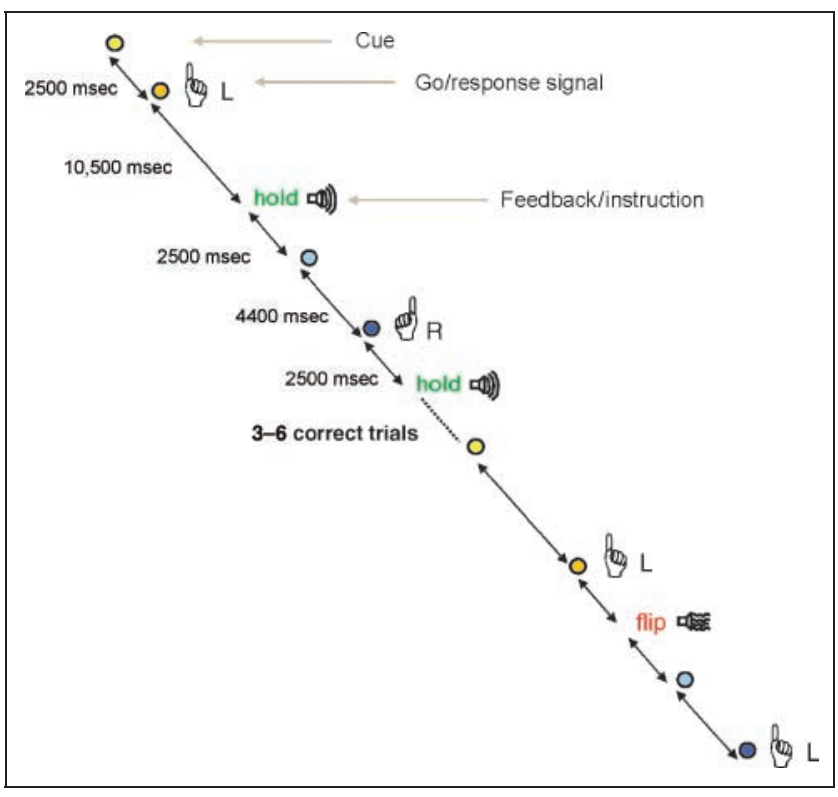

Figure 1. Schematic of experimental task. In Experiment 1, subjects associated a colored cue with a left/right button press. Dimming of the cue acted as a go signal. Following each response, a feedback/ instruction was presented instructing the subjects to "hold" or "flip" the rule on the following trial. Rule "flip" instructions occurred after runs of between three and six consecutive correct response trials. Variable interevent intervals ensured that unique regressors could be generated based on the predicted BOLD response to each event type. Experiment 2 was identical in all respects except that the subjects were instructed to maintain the same rule throughout the task regardless of the feedback. faces: $:(-)$ ), with half the subjects receiving words and the other half faces. Previous work had shown differences in the characteristics of behavioral switch costs using the two types of stimuli (Hodgson, Golding, Molyva, Rosenthal, \& Kennard, 2004). Other work has also suggested the existence of a gradient of processing within the medial frontal cortex, with processing of emotional/affective information taking place in more anterior regions (Bush, Luu, \& Posner, 2000). However, between-subject group $t$ tests for the event-related activity evoked for two types of feedback stimulus revealed no significantly activated voxels and the two groups were collapsed in the main fMRI analysis reported below. Both types of hold and flip rule feedbacks were accompanied by a high and low pitched tone, respectively. If the subject's response was incorrect, the word "error" would appear instead of the feedback.

Rule reversals occurred following runs of correct responses of between three and six trials in length. The period between each event was variable and was equally likely to be either 2200, 4400, or 10,200 msec. These periods were chosen to optimize the efficiency of the event-related design according to the theoretical principles outlined elsewhere (Josephs \& Henson, 1999; Dale \& Buckner, 1997). This allowed a true multievent-related design to be implemented in which activity evoked by each discrete event within a trial could be visualized. All subjects performed 105 trials in five equal runs with the first 21 trials serving as the practice trials (for which scans were not acquired).

\section{Data Analysis}

Data were analyzed using SPM2 software (www.fil.ion. ucl.ac.uk/spm). The images were realigned, unwarped to remove variance caused by movement-by-fieldinhomogeneity interactions, normalized to a standard EPI template, and smoothed with a Gaussian kernel of $6 \mathrm{~mm}$ full width at half maximum. Interevent periods were selected such that the cue, go-signal, and feedback event sequences were temporally de-correlated within a block (see Task and Procedure above), allowing unique regressors to be derived for each event type by convolving the event onset times with a canonical hemodynamic response function. The different event types modeled were: feedback hold, feedback flip, cue bold, cue flip, go bold, and go flip (for cue and go events, "flip" trials correspond to events immediately following presentation of a flip feedback). A general linear model approach was used to estimate parameter values for each regressor and a series of one-sample $t$ tests were carried out to determine whether the fitted parameter values at each voxel for each subject were significantly greater than zero for each modeled event. This generated a series of " $t$ contrast images" for each effect and subject, which were entered into a secondlevel ("random effects") analysis to test which voxels 
showed consistent "activation" across subjects. This analysis used one-sample $t$ tests (for the main effect of each event type) and two-sample repeated-measures $t$ tests (for contrasts between event types) with a false discovery rate (FDR) corrected statistical threshold of $p<.05$ and a voxel cluster size threshold of 12 . Finally, for the between-subject groups comparison of Experiments 1 and 2 (see below), two-sample unrelated $t$ tests were used, uncorrected for multiple comparisons. The $x, y, z$ coordinates of all activation clusters were transformed from normalized MNI space (i.e., SPM coordinates) to Talairach space (www.mrc-cbu.cam.ac. uk/Imaging/mnispace.html) in order to ascertain the site of activation relative to the atlas of Talairach and Tournoux (1998).

\section{Results}

\section{Behavioral Data}

Reaction time and error rates were analyzed relative to the occurrence of rule reversals. A significant reduction in mean reaction time to the go signal (dimming of the cue) was observed following a reversal in SR mappings
(Trial 1 after rule reversal: $505 \pm 128 \mathrm{msec}$; Trial 2: $545 \pm$ $134 \mathrm{msec}$ ). Error rates were very low (averaging 3\% across all subjects/trials) and did not vary significantly relative to the occurrence of rule reversals.

\section{fMRI Data}

Feedback Onset Events. A one-sample $t$ test (random effects, FDR correction, $p<.05$ ) for "flip" feedback events (i.e., all activity correlated with event onset relative to null) revealed widespread activity in lateral and medial prefrontal regions. Areas showing a blood oxygen level dependent (BOLD) signal, which significantly correlated with "flip" feedbacks, included areas of the ventral (BA 37) and dorsal (BA 46) lateral frontal cortex as well as the anterior cingulate gyrus (BA 32) (Talairach coordinates: $-2,19,38)$. The equivalent analysis for "hold" events revealed no significant activity in prefrontal regions (Figure 2).

The repeated-measures contrast between "hold" and "flip" feedback events also showed significantly increased activity for "flip" relative to "hold" feedbacks in a number of regions of the prefrontal cortex, including the anterior cingulate, as well as dorsolateral

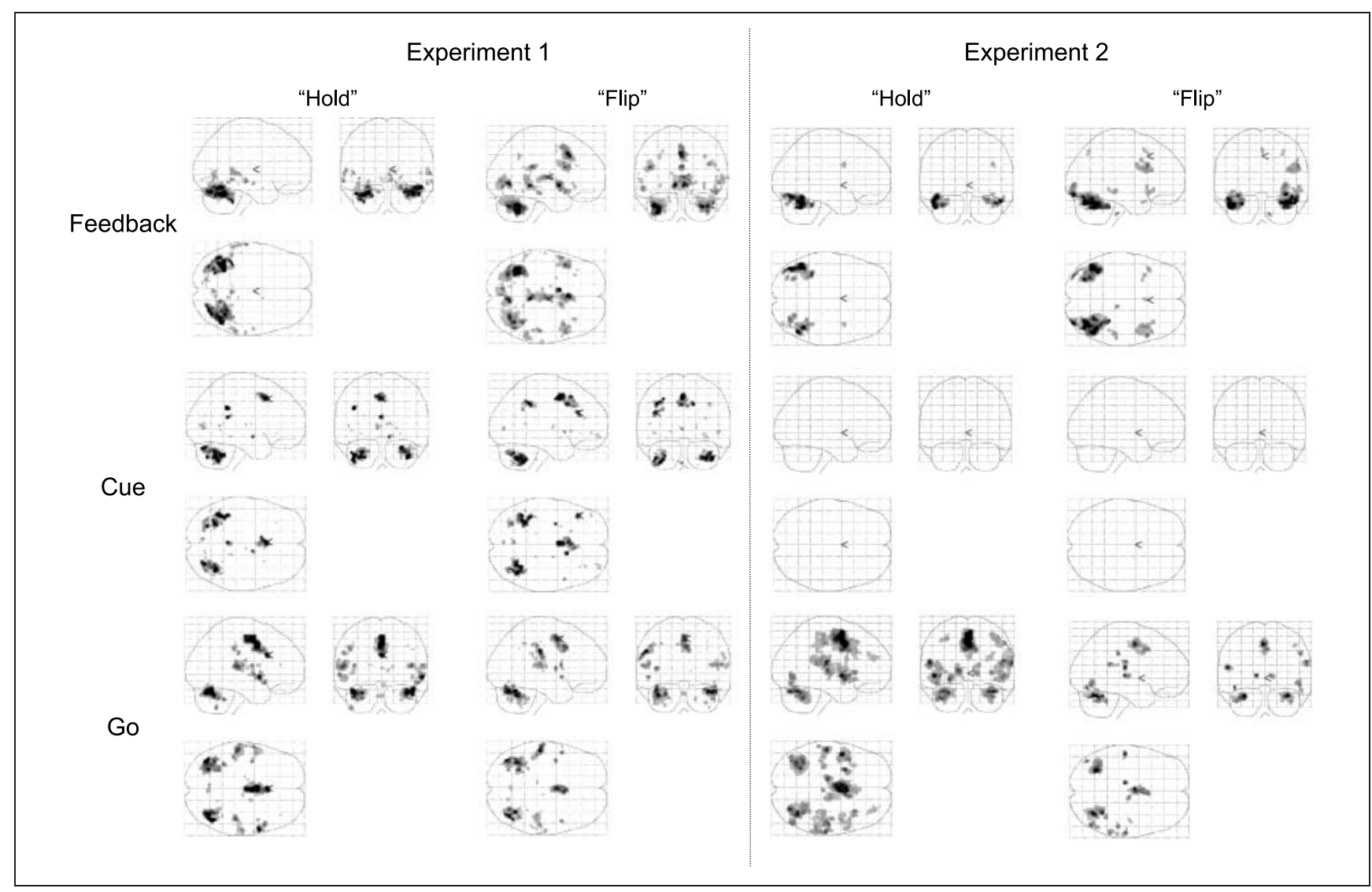

Figure 2. Event-related activations for the three discrete events types (feedback, cue, and go) for "hold" and "flip" trials in Experiments 1 and 2 (for cue and go events, "flip" trials correspond to events immediately following a flip feedback). Main effects (relative to null) are shown in the glass brain views (random effects analysis, one-sample $t$ tests, FDR correction, $p<.05$ ). 
and ventrolateral regions. A strong subcortical locus of activation was also seen in the mediodorsal nucleus of the thalamus. Other regions outside the prefrontal cortex were also found to be significantly more active for "flip" relative to "hold" feedbacks. These included the inferior parietal lobule and the lateral cerebellum (Table 1).

Cue Onset Events. One-sample $t$ tests for cue events immediately following either "flip" or "hold" feedbacks revealed activity in the medial prefrontal cortex and the lateral cerebellum for both cue event types (Figure 2). However, the locus of medial activation was more posterior than that observed for feedback events (see above), corresponding to activity in BA 24 (Talairach coordinates: $-2,2,42)$. A significant cluster of activation was also present in the left dorsolateral prefrontal cortex (BA 46) (-41, 28, 22) for cue onsets on "flip" but not "hold" trials.
Go Signal Events. One-sample $t$ tests (FDR corrected, $p<.05)$ were also carried out to examine activity in response to go-signal events that followed the presentation of either "flip" or "hold" feedbacks. Activity was observed in the medial frontal cortex, including a locus within BA 24 on "flip" trials and regions of both BA 24 and BA 6 on "hold" trials. Significant activity was observed in the lateral cerebellum under both conditions, and bilateral activity in inferior parietal and superior temporal lobe regions was also present (Figure 2).

\section{Discussion}

Experiment 1 revealed a number of brain regions that showed an increased BOLD signal for "flip" compared with "hold" trials. The most marked difference between the two trial types was for activity synchronized to the feedback events, which instructed the maintenance or

Table 1. "Flip" Minus "Hold" Feedback Event, Repeated Measures Contrast for Experiment 1

\begin{tabular}{|c|c|c|c|c|c|c|c|c|c|c|c|}
\hline \multicolumn{6}{|c|}{ Left Hemisphere } & \multicolumn{6}{|c|}{ Right Hemisphere } \\
\hline \multirow{2}{*}{$\frac{\text { Clusters }}{\text { Frontal }}$} & \multirow[t]{2}{*}{$Z$} & \multicolumn{3}{|c|}{ Talairach } & \multirow[t]{2}{*}{ Anatomy } & \multirow[t]{2}{*}{ Clusters } & \multirow[t]{2}{*}{$Z$} & \multicolumn{3}{|c|}{ Talairach } & Anatomy \\
\hline & & & & & & & & & & & \\
\hline 427 & 5.02 & -2 & 19 & 37 & Anterior cingulate (BA 32) & & & & & & \\
\hline \multirow[t]{3}{*}{13} & 3.85 & -30 & 26 & -4 & Inferior frontal gyrus (BA 47) & 104 & 4.44 & 44 & 18 & -10 & Inferior frontal gyrus (BA 47) \\
\hline & & & & & & 16 & 4.06 & 53 & 18 & & (BA 45) \\
\hline & & & & & & 40 & 3.96 & 34 & 27 & -8 & (BA 47) \\
\hline 143 & 4.07 & -42 & 20 & 21 & Middle frontal gyrus (BA 46) & & & & & & \\
\hline 33 & 4.40 & -26 & -2 & 41 & & & & & & & \\
\hline 11 & 3.89 & -38 & 30 & 20 & & & & & & & \\
\hline \multicolumn{12}{|c|}{ Occipital, Temporal, Parietal } \\
\hline 67 & 4.28 & -56 & 10 & -8 & Superior temporal gyrus (BA 22) & & & & & & \\
\hline 29 & 4.22 & -34 & -50 & 36 & Inferior parietal lobe (BA 40) & & & & & & \\
\hline 15 & 3.88 & -2 & -78 & 4 & Lingual gyrus (BA 18) & & & & & & \\
\hline 63 & 4.58 & -1 & -32 & 22 & Posterior cingulate gyrus (BA 23) & & & & & & \\
\hline \multicolumn{12}{|c|}{ Subcortical } \\
\hline 275 & 5.71 & -34 & $-52-$ & -42 & Cerebellum, posterior lobe & 178 & 4.62 & 38 & -50 & -31 & Cerebellum, posterior lobe \\
\hline \multirow[t]{4}{*}{39} & 3.93 & -32 & $-69-$ & -25 & & & & & & & \\
\hline & & & & & & 48 & 4.88 & 12 & 10 & 0 & Right head of caudate \\
\hline & & & & & & 9 & 4.06 & 16 & 19 & 1 & Right head of caudate \\
\hline & & & & & & 87 & 5.54 & -16 & 12 & -4 & Right putamen \\
\hline 15 & 3.76 & -10 & -18 & 10 & Mediodorsal thalamus & 125 & 4.42 & 2 & -8 & 8 & Mediodorsal thalamus \\
\hline
\end{tabular}

Peak activations for clusters greater than $500 \mathrm{~mm}^{3}, 2 \times 2 \times 2$ FDR corrected, $p<.05$. 
reversal of the current SR association (Figure 2; Table 1). Significantly increased prefrontal activity was observed during presentation of "flip" relative to "hold" feedbacks. This included significant loci within the bilateral dorsal and ventrolateral prefrontal cortex, as well as a strong signal within the anterior cingulate and the mediodorsal nucleus of the thalamus.

The presence of activity in the anterior cingulate gyrus following "flip" feedbacks is consistent with previous studies which have implicated this region in the control of rule/task switching (Bush, et al., 2002; Rushworth et al., 2002). In the present study, we also found that the anterior cingulate activation was accompanied by a strong subcortical signal within the mediodorsal nucleus of the thalamus. This part of the thalamus is known to send extensive connections to medial prefrontal regions. For example, one recent study has shown that direct electrical stimulation of this structure in the rat causes an increase in BOLD response within the ipsilateral anterior cingulate (Shyu et al., 2004). The mediodorsal thalamus also receives input from the anterior cingulate, as well as the lateral prefrontal cortex and amygdala, and forms part of the fronto-striatal system of reciprocal cortical-subcortical loops (Alexander, DeLong, \& Strick, 1996). Consistent with coherent activation of corticostriatal loops during rule changes, we also observed activity within the striatum (head of caudate/putamen) following "flip" feedbacks.

The medial prefrontal cortex comprises multiple anatomical regions of which the anterior cingulate region is just one. The organizational principles underlying this network of areas has been of considerable interest to researchers over recent years. In the present study, medial frontal activity was observed for cue, go, and feedback events. However, the locus of this activity shifted during the course of the trial, with "flip" feedbacks activating the most anterior regions, followed by cue events (pre-SMA) and go-signal onsets (SMA proper).

In order to examine the statistical significance of these shifts in activity between the different event types, we performed a post hoc analysis of peak activity in the medial frontal cortex for individual subjects for each event type. The coordinates of peak activation clusters lying within the medial frontal cortex were noted for each event type and individual subject. The $x, y$, $z$ coordinates of these activation loci were entered into repeated-measures $t$ tests to determine whether there was a consistent shift in the locus of medial prefrontal activity between event types across subjects.

This analysis supported the findings of the random effects analysis and confirmed that there was a consistent organization within medial prefrontal cortical areas across subjects. The mean locus of activity evoked by feedback events was found to shift anteriorly (i.e., along the $y$-axis) relative to cue and go event activations $(t=2.58, p<.025)$. Cue event activity was, in turn, more anterior compared to that evoked by the go-signal $(t=3.05, p<.005)$ (Figure 3). This analysis is consistent with the existence of a functional gradient within the frontal medial wall moving from more cognitive functions anteriorly, through motor preparatory to motor output generation within posterior regions.

Activation was also observed in ventral and dorsal regions of the lateral prefrontal cortex. Activity in these areas following the "flip" feedback/instruction can be interpreted as reflecting a number of possible neurocognitive operations. For example, it may represent the updating of task rules within working memory or the imposition of enhanced top-down control in readiness for increased response conflict (Aron et al., 2004; Swainson et al., 2003; MacDonald et al., 2001; Konishi, Kawazua, et al., 1999). However, a potential confound in the design of Experiment 1 makes it difficult to interpret prefrontal activity following "flip" events as being exclusively related to the demands of reversing SR associations. Rule changes only occurred after three to six consecutive correct responses. Because of this, there were a greater number of "hold" trials relative to "flip" trials (by a ratio of approximately 3:1). Activity correlated with "flip" feedbacks may therefore reflect the occurrence of an "oddball" stimulus, regardless of its behavioral significance. Indeed, such an explanation for the observed lateral frontal activation in our study would be consistent with previous work that has shown activity in this region during the processing of "surprising" and infrequent events (Braver et al., 2001; Fletcher et al., 2001).

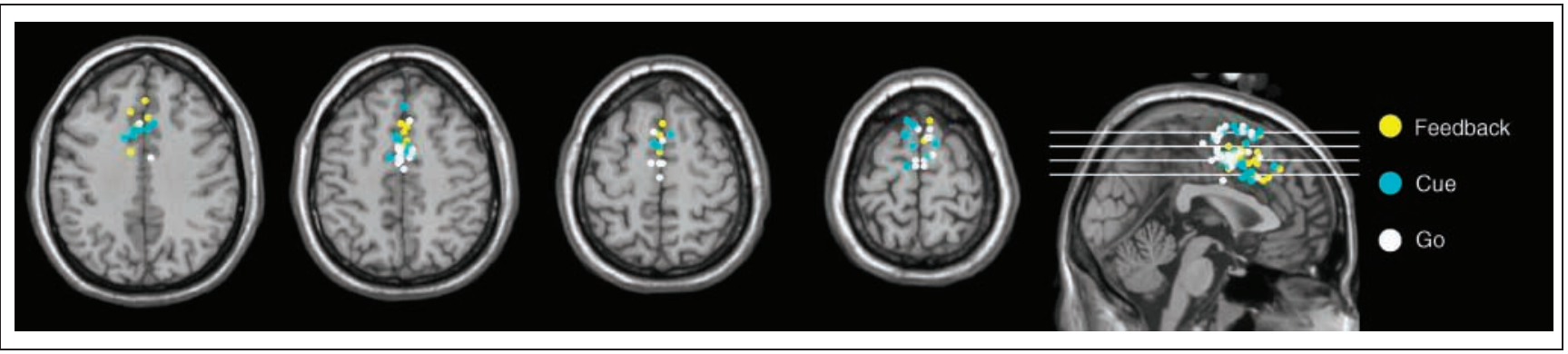

Figure 3. The coordinates of peak activation clusters lying within the medial frontal cortex for individual subjects in Experiment 1 . A consistent anterior to posterior shift in activity was observed from feedback, cue through to go events (see Discussion Experiment 1 for details). 
In order to assess whether activity associated with rule change instructions was due to the distinct perceptual characteristics of "flip" events rather than the demands of reconfiguring SR mappings, we carried out a second experiment using almost exactly the same stimulus and procedure with a new group of subjects. However, in this version of the task, participants were instructed to hold the same SR mappings throughout the experiment regardless of the feedback given after each response. The ratio of "hold" to "flip" feedbacks was kept the same, all that was modified was the instructions given to the subject, such that in Experiment 2 "flip" feedbacks did not have any task significance. Matching the design in this manner allowed a direct comparison to be made between the pattern of brain activity in Experiments 1 and 2 .

\section{EXPERIMENT 2}

\section{Methods}

Twenty-two right-handed volunteers participated in Experiment 2, ranging in age from 18 to 39 years (mean age $=25$ years). Five were men and 17 were women, and none had taken part in Experiment 1. Methods were identical in all respect relative to Experiment 1, except that the rules linking stimulus and response remained constant throughout. "Flip" feedback events occurred with the same likelihood and frequency as in Experiment 1 , but participants were told that the feedback was irrelevant to the response to be made and the rule linking color with response would remain constant throughout.

\section{Results}

\section{Behavioral Data}

Mean reaction times did not vary significantly with the relative position of the trial after the occurrence of a "flip" cue (Trial 1 after rule reversal: $504 \pm 124$ msec; Trial 2: $518 \pm 161 \mathrm{msec})$. Similarly, error rates did not vary systematically across trials (averaging 1\% of all subjects/trials).

\section{fMRI Data}

Feedback, Cue, and Go Signal Events. Using the same analysis procedure as Experiment 1, one-sample $t$ tests examined which areas showed a BOLD response that was significantly correlated with the occurrence of feedback, cue, and go events for "flip" and "hold" rule trials (we refer to these event types as "flip" and "hold" for convenience even though they were not associated with rule changes in this experiment) (see Figure 2).

Surprisingly, the distribution of activity for feedback events was very similar to that observed in Experiment 1.
Regions activated by so-called flip feedbacks in Experiment 2 included the right dorsolateral prefrontal cortex, BA 9/46 (Talairach coordinates of peak activation clusters: 52, 19, 25 and 46, 25, 19); the left ventrolateral prefrontal cortex, BA $13(-44,20,8)$; and the medial frontal cortex, BA $6(0,14,47)$. This was despite the fact that these stimulus events had no bearing on task performance. In contrast to Experiment 1, none of the repeated measures comparisons revealed any voxels which were significantly more active for "flip" relative to "hold" trial events in Experiment 2.

Comparison with Experiment 1. In order to test which of the areas activated by feedback events in Experiment 1 were related to the requirement to reverse rules, rather than reflecting the perceptual characteristics of the "flip" feedbacks, a direct comparison was carried out between activity for "flip" feedbacks in Experiment 1 versus Experiment 2. This revealed three significant loci of activation: the mediodorsal nucleus of the thalamus, the anterior cingulate gyrus, and the left lateral cerebellum (unrelated two-sample $t$ test, uncorrected for multiple comparisons, $p<.001$, cluster threshold $=$ 12) (Figure $4 \mathrm{~A}$ ). Importantly, regions of the ventrolateral and dorsolateral frontal cortex failed to show any significant increase in activity for Experiment 1 relative to Experiment 2. Full details of this contrast are given in Table 2. The equivalent between-groups comparison for "hold" feedback events showed only a single cluster of voxels in the anterior cingulate gyrus that was significantly more active in Experiment 1 relative to Experiment 2 (BA 32: 0, 9, 35) (Figure 4B).

For completeness, we also carried out the reverse comparison to determine whether any regions showed increased activity for "flip" and "hold" events in Experiment 2 relative to Experiment 1. This analysis revealed no significant changes in activity in the medial/lateral frontal regions or the dorsomedial thalamus.

\section{GENERAL DISCUSSION}

The only difference between Experiments 1 and 2 was the instructions given to subjects. Participants were instructed to periodically reverse SR associations in response to the "flip" instruction in Experiment 1, but to maintain the same SR associations regardless of the feedbacks in Experiment 2. We assume therefore that brain regions showing significant changes in activity between the two experiments are recruited by the demands of maintaining and manipulating arbitrary rules and SR mappings.

When a direct statistical comparison was made to test which brain regions showed increased activity for "flip" feedback events in Experiment 1 relative to Experiment 2, activation clusters were only present in 


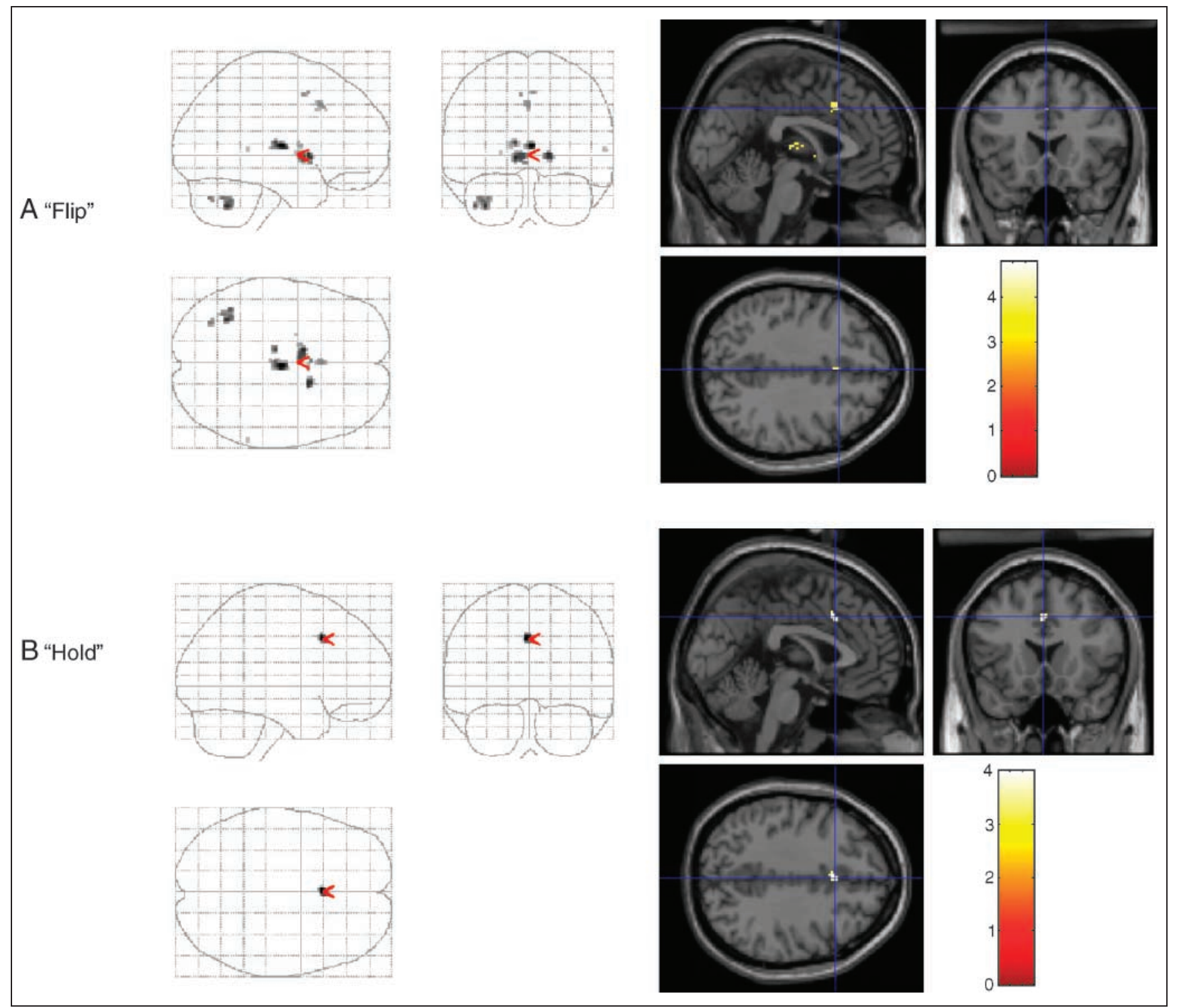

Figure 4. Glass brain and sectional view of areas showing increased activity in Experiment 1 relative to Experiment 2 for (A) "flip" feedback events and (B) "hold" feedback events (two-sample between-groups $t$ test, $p<.001$, uncorrected for multiple comparisons, intensity plot on sectional view indicate $t$ scores).

the anterior cingulate, mediodorsal thalamus, putamen, and left cerebellum (Table 2; Figure 4A). Other regions, including the dorsolateral and ventrolateral prefrontal cortex, showed no significant difference in their re- sponse to "flip" feedbacks regardless of the demand to change rules.

This result is important because it implies a dissociation of function between lateral and medial prefrontal

Table 2. Voxels Showing Greater Activity for "Flip" Feedback Events in Experiment 1 Relative to Experiment 2

\begin{tabular}{|c|c|c|c|c|c|c|c|}
\hline \multicolumn{4}{|c|}{ Left Hemisphere } & \multicolumn{4}{|c|}{ Right Hemisphere } \\
\hline Clusters & $Z$ & Talairach & Anatomy & Clusters & $Z$ & Talairach & Anatomy \\
\hline 41 & 4.01 & $\begin{array}{lll}-10 & 4 & -2\end{array}$ & Putamen & 41 & 4.30 & $2-138$ & Mediodorsal thalamus \\
\hline 47 & 3.93 & $-34-55-31$ & Cerebellum, posterior lobe & 20 & 4.37 & 80 & Putamen \\
\hline 14 & 3.48 & $\begin{array}{lll}-2 & 18 & 40\end{array}$ & Anterior cingulate (BA 32) & & & & \\
\hline
\end{tabular}

Between-subject groups, two-sample $t$ test (uncorrected, $p<.001$ ). Peak activations for clusters greater than $500 \mathrm{~mm}^{3}$. 
regions during rule reversals. Lateral frontal areas are activated by the occurrence of attentionally salient or surprising events, in this case, the "flip" feedback. However, these areas do not show significantly increased activity when this feedback indicates that SR associations need to be reversed. In contrast, activity in the anterior cingulate gyrus and associated subcortical structures was found to be increased when the feedback indicated that action needed to be taken.

Interestingly, the relative increase in anterior cingulate activity for Experiment 1 compared to Experiment 2 was not confined to "flip" feedback events but was also apparent on "hold" trials (Figure 4B). Superficially, this result is hard to reconcile with the analysis of the eventlocked activity for "hold" feedbacks in Experiment 1 (relative to null), which produced no significant activation (Figure 2). However, this pattern of significant activity in the blockwise contrast (between experiments), in the absence of transient event-locked activity in Experiment 1, suggests that as well as a phasic increase in activity when SR associations have to be modified, the anterior cingulate also shows a tonic increase in activity under conditions where SR associations are labile.

In contrast, some areas did show activity which was exclusively correlated with instructions to reverse SR mappings. However, all of these clusters were located within subcortical structures. These included the mediodorsal thalamus and other components of corticostriatal loops (i.e., the striatum and basal ganglia) as well as the lateral cerebellum (Table 2; Figure 4A). It has been proposed that the neural representation of task schema may be instantiated within the matrix of synaptic weightings in subcortical and posterior brain structures, whereas frontal regions play a role in monitoring and updating these mappings (e.g., Miller \& Cohen, 2001). The finding that only subcortical structures are exclusively activated by SR reversals is consistent with this hypothesis.

Taken together, the results can also be seen as consistent with a functional neuroanatomical prefrontal model similar to that proposed by Gehring and Knight (2000). We suggest that lateral prefrontal regions respond to attentionally salient/surprising sensory events and evaluate whether such events indicate that modification to current behavioral rules is required. When such an event signals that a change in SR mappings is required, the anterior cingulate and subcortical areas, such as the mediodorsal thalamus and striatum, show an increase in activity correlated with the actual modification of SR mappings. Under conditions for which frequent changes occur in transformations between stimulus and response, tonic activity in the anterior cingulate gyrus is observed, perhaps reflecting sustained control/monitoring of SR associations.

In order to test this account further, we examined whether activity evoked by flip feedback events varied between individual subjects dependent upon the accu- racy with which they performed the task in Experiment 1 (Figure 5). This analysis revealed strong positive correlations between the peak event-related response (i.e., the magnitude of the fitted hemodynamic response function) and the rate of response errors within the left dorsolateral, right ventrolateral, and dorsal anterior cingulate regions. Interestingly, however, no significant correlation was found within the mediodorsal nucleus of the thalamus. Furthermore, direct statistical contrasts between the group mean activity in these regions for subjects who either made at least one response error compared with those who performed the task errorfree, revealed a significant reduction in the magnitude of the event-related response within the mediodorsal thalamus for error-prone subjects (Wilcoxon $W=59.0$, $p<.025)$, with a trend towards an increase in activity in other regions.

This pattern of variation in activity with error rates can be viewed as lending support to the model of frontal organization outlined above. Both lateral and medial frontal activity appears to be related to general attentional demands imposed upon subjects by the task (increased activity being apparent in subjects that find the task more demanding). In contrast, only activity in subcortical structures (i.e., the mediodorsal thalamus) was correlated with efficient updating of SR associations (reduced activity being associated with failures to update these mappings).

It is important to note that a number of previous studies of task/rule switching have reported lateral frontal activity correlated with task changes, even when the ratio between switch and nonswitch trials is 50\% compared to the $33 \%$ likelihood used in the present research (Brass et al., 2003; Braver et al., 2003; Swainson et al., 2003; Luks, Simpson, FeiWell, \& Miller, 2002). Only one previous study to our knowledge has systematically examined how frontal activity varies with stimulus frequency. Braver et al. (2001) found that bilateral ventral and dorsal prefrontal regions were indeed selectively activated by oddball perceptual events. However, the demand to inhibit motor responding recruited additional activity with ventral and dorsolateral frontal regions within the right (but not left) hemisphere.

In future work, we plan to systematically vary the proportion of flip to hold rule trials. A particularly interesting test for the perceptual novelty explanation of lateral frontal activations would be the case for which flip trials occur more frequently than hold trials. Under these conditions, our account makes a specific and surprising prediction: Medial frontal and subcortical structures, such as the dorsomedial thalamus, should still be selectively activated by changes in SR mappings (i.e., switch trials). However, the left dorsolateral prefrontal cortex should actually show a stronger response for hold rule trials.

In addition to its application to the simple task studied here, the model of the prefrontal cortex emerging from 


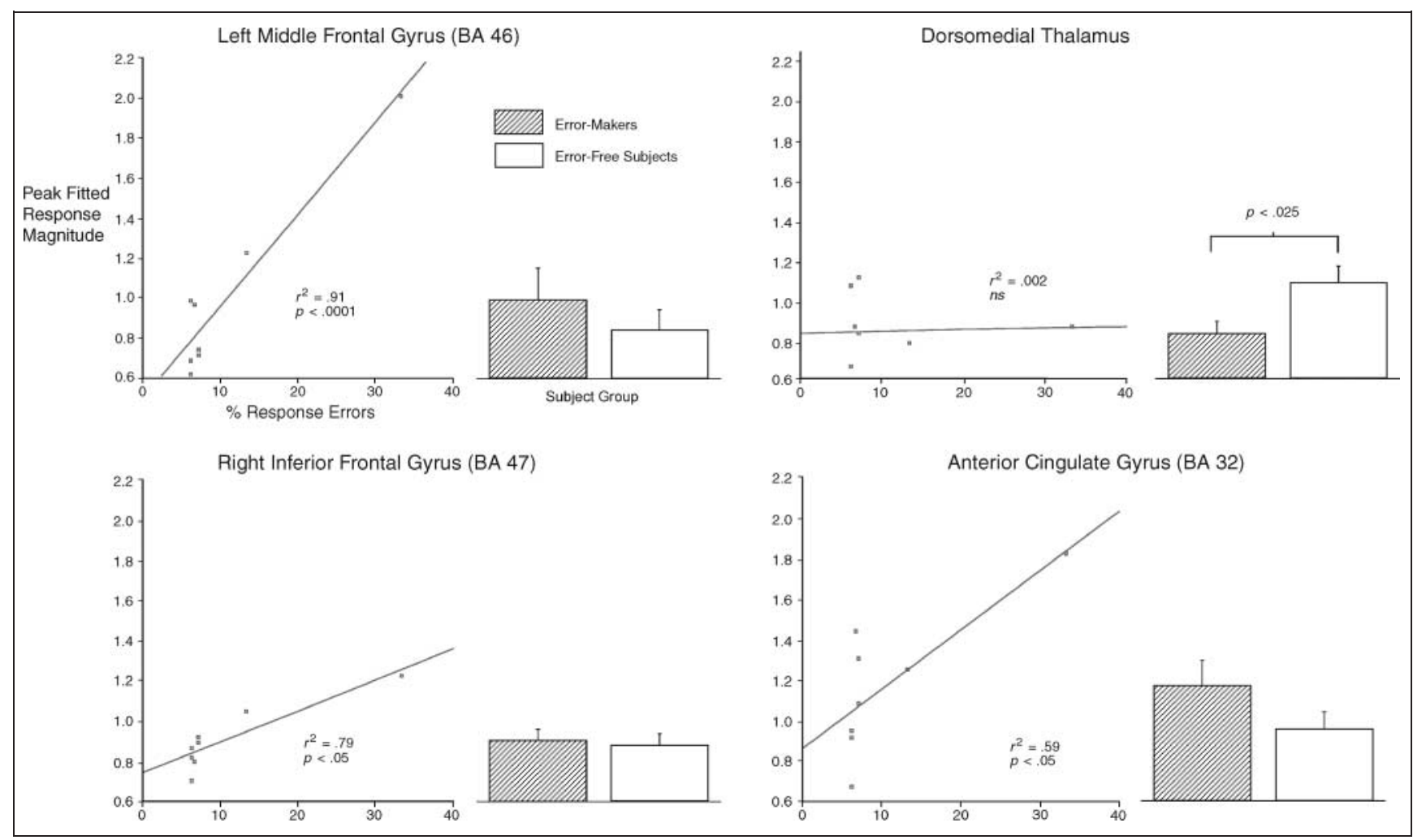

Figure 5. Relationship between individual subject performance and peak event-related response to "flip" feedback events in Experiment 1 (Talairach coordinates for locations tested were derived from the coordinates of peak activations shown in Table 1). Scatterplots show correlation between activation in each region and the occurrence of response errors on trials immediately following presentation of a flip feedback instruction. Bar plots show mean event-related response for subjects who made either no response errors (error-free subjects) compared with those who made at least one such error (error makers).

this and other studies of task switching is applicable to a range of superficially diverse tasks (Duncan \& Owen, 2000). All these tasks require the active monitoring and evaluation of sensory events for the purpose of optimizing nonstandard transformations between sensory input and motor output. Efficient implementation of these control operations essentially requires the temporal integration of sensory and motor signals across delays. The neural architecture of prefrontal regions provides a suitable substrate for such a working memory system in the form of sustained neural activity within reciprocal cortico-cortical and cortical-subcortical loops (Fuster, 2000; Goldman Rakic, 1995). Failure to maintain a trace of past actions and their associated sensory context would lead to deficits in updating and maintaining SR associations, similar to the type of deficit seen following frontal lobe damage in humans and animals.

\section{Conclusions}

We have used a very simple model task to investigate the contributions of various prefrontal regions to the adaptive control of behavior. The results clarify the relative specializations of the medial and lateral prefrontal cortex during switching between arbitrary task rules. Ventral and dorsal lateral prefrontal areas are recruited by behaviorally salient/surprising sensory events regardless of their behavioral implications. When such events signal the necessity to modify current behavior, the anterior cingulate cortex and associated subcortical structures, including the mediodorsal thalamus, are recruited to monitor and adjust SR mappings.

\section{Acknowledgments}

This research was supported by Wellcome Trust Project Grant No. 072082/Z/03/Z. We thank two anonymous reviewers for providing helpful comments on the manuscript and offering useful suggestions for additional data analysis.

Reprint requests should be sent to Timothy L. Hodgson, Exeter Centre for Cognitive Neuroscience, School of Psychology, University of Exeter, Exeter, UK, or via e-mail: t.l.hodgson@ ex.ac.uk.

\section{REFERENCES}

Alexander, G. E., DeLong, M., \& Strick, P. L. (1996). Parallel organization of functionally segregated circuits linking basal ganglia and cortex. Annual Review of Neuroscience, 9, 357-381.

Aron, A. R., Monsell, S., Sahakian, B. J., \& Robbins, T. W. (2004). A componential analysis of task-switching deficits associated with lesions of left and right frontal cortex. Brain, 127, 1561-1573. 
Asaad, W. F., Rainer, G., \& Miller, E. K. (1998). Neural activity in the primate prefrontal cortex during associative learning. Neuron, 21, 1399-1407.

Baddeley, A. (1988). Working memory. Oxford, UK: Oxford University Press.

Barbas, H., \& De Olmos, J. (1990). Projections from the amygdala to basoventral and mediodorsal prefrontal regions in the rhesus monkey. Journal of Comparative Neurology, $301,1-23$.

Barbas, H., Henion, T. H., \& Dermon, C. R. (1991). Diverse thalamic projections to the prefrontal cortex in the rhesus monkey. Journal of Comparative Neurology, 313, 65-94.

Barbas, H., \& Pandya, D. N. (1989). Architecture and intrinsic connections of the prefrontal cortex in the rhesus monkey. Journal of Comparative Neurology, 286, 353-375.

Brass, M., Ruge, H., Meiran, N., Rubin, O., Koch, I., Zysset, S., et al. (2003). When the same response has different meanings: Recoding the response meaning in the lateral prefrontal cortex. Neuroimage, 20, 1026-1031.

Braver, T. S., Barch, D. M., Gray, J. R., Molfese, D. L., \& Snyder, A. (2001). Anterior cingulate cortex and response conflict: Effects of frequency, inhibition and errors. Cerebral Cortex, 11, 825-836.

Braver, T. S., Reynolds, T. R., \& Donaldson, D. I. (2003). Neural mechanisms of transient and sustained cognitive control during task switching. Neuron, 39, 713-726.

Bush, G., Luu, P., \& Posner, M. I. (2000). Cognitive and emotional influences in anterior cingulate cortex. Trends in Cognitive Sciences, 4, 215-222.

Bush, G., Vogt, B. A., Holmes, J., Dale, A. M., Greve, D., Jenike, M. A., et al. (2002). Dorsal anterior cingulate cortex: A role in reward-based decision making. Proceedings of the National Academy of Sciences, U.S.A., 99, 523-528.

Carter, C. S., Botvinick, M. M., \& Cohen, J. D. (1999). The contribution of the anterior cingulate cortex to executive processes in cognition. Reviews in the Neurosciences, 10, 49-57.

Cohen, J. D., \& Servan-Schreiber, D. (1992). Context, cortex, and dopamine: A connectionist approach to behavior and biology in schizophrenia. Psychological Review, 99, 45-77.

Dale, A. M., \& Buckner, R. L. (1997). Selective averaging of rapidly presented individual trials using fMRI. Human Brain Mapping, 5, 329-340.

Derrfuss J., Brass, M., \& von Cramon, Y. (2004). Cognitive control in the posterior fronto-lateral cortex: Evidence from common activations in task coordination, interference control, and working memory. Neuroimage, 23, 604-612.

D'Esposito, M., Aguirre, G. K., Zarahn, E., Ballard, D., Shin, R. K., Lease, J., et al. (1998). Functional MRI studies of spatial and nonspatial working memory. Cognitive Brain Research, 7, 1-13.

Duncan, J. (1999). Converging levels of analysis in the cognitive neuroscience of visual attention. In G. W. Humphreys, J. Duncan, \& A. Triesman (Eds.), Attention, space and action (pp. 112-129). Oxford, UK: Oxford University Press.

Duncan, J., \& Owen, A. M. (2000). Common regions of the human frontal lobe recruited by diverse cognitive demands. Trends in Cognitive Sciences, 23, 475-483.

Fletcher, P. C., Anderson, J. M., Shanks, D. R., Honey, R., Carpenter, T. A., Donovan, T., et al. (2001). Responses of human frontal cortex to surprising events are predicted by formal associative learning theory. Nature Neuroscience, 4 , $1043-1048$

Forstmann, B. U., Brass, M., Koch, I., \& Yves von Cramon, D. (2005). Internally generated and directly cued task sets: An investigation with fMRI. Neuropsychologia, 43, 943-952
Fuster, J. M. (2000). Executive frontal functions. Experimental Brain Research, 133, 66-70

Gehring, W. J., \& Knight, R. T. (2000). Prefrontal-cingulate interactions in action monitoring. Nature Neuroscience, 3, 516-520.

Goldman-Rakic, P. S. (1995). Architecture of the prefrontal and the central executive. Proceedings of the National Academy of Sciences, U.S.A., 769, 71-83.

Hodgson, T. L., \& Golding, C. (2003). Executive contributions to eye movement control. In J. Hyona, R. Radach, \& H. Deubel (Eds.), The mind's eye: Cognitive and applied aspects of eye movement research (pp. 49-64). Amsterdam: North Holland-Elsevier Science.

Hodgson T. L., Golding C., Molyva D., Rosenthal C. R., \& Kennard C. (2004). Eye movements during task switching: Reflexive, symbolic, and affective contributions to response selection. Journal of Cognitive Neuroscience, 16, 318-330.

Husain, M., Parton, A., Hodgson, T., Mort, D., \& Rees, G. (2003). Self-control during response conflict by human supplementary eye field. Nature Neuroscience, 6, 117-118.

Josephs, O., \& Henson, R. N. A. (1999). Event-related functional magnetic resonance imaging: Modelling, inference and optimization. Philosophical Transactions of the Royal Society of London, Series B, Biological Sciences, 354, 1215-1228.

Kimberg, D. Y., Aguirre, G. K., \& D'Esposito, M. (2000). Modulation task-related neural activity in task-switching: An fMRI study. Cognitive Brain Research, 10, 189-196.

Konishi, S., Hayashi, T., Uchida, I., Kikyo, H., Takahashi, E., \& Miyashita, Y. (2002). Hemispheric asymmetry in human lateral prefrontal cortex during cognitive set shifting. Proceedings of the National Academy of Sciences, U.S.A., 99, 7803-7808.

Konishi, S., Kawazua, M., Uchida, I., Kikyo, H., Asakura, I., \& Miyashita, Y. (1999). Contribution of working memory to transient activation in human inferior prefrontal cortex during performance of the Wisconsin Card Sorting Task. Cerebral Cortex, 9, 745-753.

Konishi, S., Nakajima, K., Uchida, I., Kikyo, H., Kameyama, M., \& Miyashita, Y. (1999). Common inhibitory mechanism in human inferior prefrontal cortex revealed by event-related functional MRI. Brain, 122, 981-991.

Kunishio, K., \& Haber, S. (1994). Primate cingulostriatal projection: Limbic striatal versus sensorimotor striatal input. Journal of Comparative Neurology, 350, 337-356.

Luks, T. L., Simpson, G. V., Feiwell, R. J., \& Miller, W. J. (2002). Evidence for anterior cingulate cortex involvement in monitoring preparatory attentional set. Neuroimage, 17, 792-802.

MacDonald, A. W., Cohen, J. D., Stenger, V. A., \& Carter, C. S. (2000). Dissociating the role of the dorsolateral prefrontal and anterior cingulate cortex in cognitive control. Science, 288, 1835-1838.

Miller, E. K., \& Cohen, J. D. (2001). An integrative theory of prefrontal function. Annual Review of Neuroscience, 24, 167-202.

Morecraft, R. J., \& Van Hoesen, G. W. (1998). Convergence of limbic input to the cingulate motor cortex in the rhesus monkey. Brain Research Bulletin, 45, 209-232.

Montaron, M. F., \& Buser, P. (1988). Relationships between nucleus medialis dorsalis, pericruciate cortex, ventral tegmental area and nucleus accumbens in cat: An electrophysiological study. Experimental Brain Research, 69, 559-566.

Owen, A. M. (2000). The role of the lateral frontal cortex in mnemonic processing: The contribution of functional neuroimaging. Experimental Brain Research, 133, 33-43. 
Passingham, R. E., Toni, I., \& Rushworth, M. F. S. (2000) Specialisation within the prefrontal cortex: The ventral prefrontal cortex and associative learning. Experimental Brain Research, 133, 103-113.

Paus, T. (2001). Primate anterior cingulate cortex: Where motor control, drive and cognition interface. Nature Reviews Neuroscience, 2, 417-424.

Rushworth, M. F. S., Hadland, K. A., Paus, T., \& Sipila, P. K. (2002). Role of the human medial frontal cortex in task switching: A combined fMRI and TMS study. Journal of Neurophysiology, 87, 2577-2592.

Sakai, K., \& Passingham, R. E. (2003). Prefrontal interactions reflect future task operations. Nature Neuroscience, 6, 75-81.

Shallice, T., \& Burgess, P. W. (1996). The domain of supervisory processes and temporal organization of behaviour. Philosophical Transactions of the Royal Society of London, Series B, 351, 1405-1412.

Shyu, B. C., Lin, C. Y., Sun, J. J., Chen, S. L., \& Chang, C. (2004). BOLD response to direct thalamic stimulation reveals a functional connection between the medial thalamus and the anterior cingulate cortex in the rat. Magnetic Resonance in Medicine, 52, 47-55.

Swainson, R., Cunnington, R., Jackson, G. M., Rorden, C., Morris, P. G., \& Jackson, S. R. (2003). Cognitive control mechanisms revealed by ERP and fMRI: Evidence from repeated task-switching. Journal of Cognitive Neuroscience, 15, 785-799.

Talairach, J., \& Tournoux, P. (1988). Co-planar stereotactic atlas of the buman brain: 3-Dimensional proportional system: An approach to cerebral imaging. Stuttgart: Thieme. 